\title{
El género Zygia P. Browne (Leguminosae, Mimosoideae, Ingeae) en la porción mexicana de la Península de Yucatán
}

\author{
José Enrique López-Contreras ${ }^{1}$, Maria de Lourdes Rico-Arce ${ }^{2}$, Lilia Lorena Can-Itza ${ }^{1} \&$ Rodrigo Duno de Stefano,* \\ ${ }^{1}$ Herbario CICY, Centro de Investigación Científica de Yucatán A.C. (CICY), Calle 43. No. 130. Col. \\ Chuburná de Hidalgo, 97200 Mérida, Yucatán, México; roduno@cicy.mx \\ ${ }^{2}$ Herbario K, Royal Botanic Gardens, Kew, Richmond, Surrey, TW9 3AB. Reino Unido
}

\begin{abstract}
Resumen
López-Contreras, J.E., Rico-Arce, M.L., Can-Itza, L.L. \& Duno de Stefano, R. 2015. El género Zygia P. Browne (Leguminosae, Mimosoideae, Ingeae) en la porción mexicana de la Península de Yucatán. Anales Jard. Bot. Madrid 72(2): e023.

En la porción mexicana de la Península de Yucatán hay tres especies del género Zygia (Leguminosae, Mimosoideae, Ingeae). Se discuten sus diferencias morfológicas y nombres correctos. Se incluye una clave, descripciones, iconografía, información ecológica, fenológica, nombres comunes y usos.
\end{abstract}

Palabras claves: Fabaceae, Flora, Mesoamerica, México.

\section{INTRODUCCIÓN}

Zygia (Mimosoideae, Ingeae) fue propuesto por P. Browne (1756) y la publicación solo incluyó una especie ("Z. arborescens, foliis ovatis... spicillatis"), que fue sinonimizada con Mimosa latifolia Linnaeus (1759); especie que Bentham (1844 y 1875) ubicó en Pithecellobium Mart. Sect. Caulanthon. En la Flora de Jamaica (Fawcett \& Rendle, 1920) se incluyó Zygia como género válido y distinto a Pithecellobium Mart. (Bentham, 1844). Los autores para formalizar el género, usan como basónimo Mimosa latifolia de Linnaeus (1759), Zygia latifolia (L.) Fawc. \& Rendle. Hoy en día, el género incluye 40-45 especies de árboles o arbustos inermes, sin braquiblastos, en su mayoría con inflorescencias caulifloras de tipo espiga o pseudoracimos, las flores son homomorfas con un disco intraestaminal rodeando la base del ovario (salvo algunas excepciones) y las semillas sin arilo con una cubierta seminal delgada sin pleurograma (Britton \& Rose, 1928; Nielsen, 1981; Barneby \& Grimes, 1997). Zygia es endémico de los trópicos y subtrópicos americanos, desde México hasta Argentina y las Antillas (Barneby \& Grimes, 1997). Varios autores no habían reconocido el género Zygia, y lo incluyeron como sinónimo de Pithecellobium (e.g. Woodson \& Schery, 1950); sin embargo otros sí llegaron a reconocer el género (Britton \& Rose, 1928; Barneby \& Grimes, 1997). Estudios recientes, basados en marcadores moleculares, confirman que Pithecellobium y Zygia son diferentes linajes. Por un lado, Z. inaequalis (Willd.) Pittier forma parte de una politomía que incluye Archidendron F. Muller, Cojoba Britton \& Rose, Inga Mill., Paraserianthes I.C. Nielsen, Pseudosamanea Harms, Zapoteca H.M. Hern., Wallaceodendron Koord. y tres alianzas con 12 géneros más (Brown \& al., 2008); otro estudio reveló usando otra especie de Zygia, que $Z$. racemosa (Ducke) Barneby \& J.W. Grimes es el grupo hermano de

\begin{abstract}
López-Contreras, J.E., Rico-Arce, M.L., Can-Itza, L.L. \& Duno de Stefano, R. 2015. The genus Zygia P. Browne (Leguminosae, Mimosoideae, Ingeae) in the Mexican portion of the Yucatan Peninsula. Anales Jard. Bot. Madrid 72(2): e023.
\end{abstract}

In the Mexican portion of the Yucatan Peninsula there are three species of Zygia (Leguminosae, Mimosoideae, Ingeae). Their morphological differences and nomenclature are discussed. A key, descriptions, iconography, ecological, phenological, uses and common names are included.

Keywords: Fabaceae, Flora, Mesoamerica, Mexico.

Macrosamanea pubiramea (Steud.) Barneby \& J.W. Grimes (Rodrigues de Souza \& al., 2013).

Actualmente existen seis nombres de Zygia citados para la porción mexicana de la Península de Yucatán (Sosa \& al., 1985; Durán \& al., 2000; Arellano \& al., 2003; Carnevali \& al., 2010). La monografía más reciente del género cita solo dos especies: Z. cognata (Schltdl.) Britton \& Rose y Z. conzattii (Standl.) Britton \& Rose (Barneby \& Grimes, 1997). Un análisis del material de herbario de México, en especial de la Península de Yucatán, Centro América y las Antillas, mostró una gran variedad morfológica; el objetivo de este trabajo fue evaluar el número de especies de Zygia que hay en la porción mexicana de la Península de Yucatán.

\section{MATERIAL Y MÉTODOS}

Para conocer qué especies de Zygia se distribuyen naturalmente en la porción mexicana de la Península de Yucatán (Campeche, Quintana Roo y Yucatán), se estudió material de México, Centro América y las Antillas proveniente de los herbarios: A, AAU, CICY, ENCB, F, G, HAL, K, MEXU, MICH, M, MO, NY, CIQRO, UCAM, US (acrónimos según Thiers, 2010). Para los morfos reconocidos se realizaron entre 15 y 25 mediciones de caracteres provenientes de hojas, flores y frutos para estimar la media, la varianza y los valores mínimos y máximos. Se realizaron comparaciones objetivas entre los morfos encontrados así como con especies de las áreas vecinas de México, Centro América y las Antillas. Las descripciones aquí presentadas solo hacen referencia a las muestras de la Península de Yucatán. La terminología utilizada es de acuerdo a Barneby \& Grimes (1997). Los mapas de distribución fueron elaborados con el programa SimpleMappr (Shorthouse, 2010). 


\section{RESULTADOS}

El análisis de material de herbario permitió reconocer tres morfoespecies en la porción mexicana de la Península de Yucatán pertenecientes al género Zygia sección Zygia que se caracteriza por sus hojas bipinnadas con un solo par de pinnas y cada pinna presenta de uno a doce pares de foliolos. La consulta de la clave de Barneby \& Grimes (1997), los tipos nomenclaturales y los protólogos de las especies, permitieron asignar estos tres morfos a: Zygia cognata, Z. latifolia var. latifolia y $Z$. recordii Britton \& Rose. La clave presentada a continuación resume las diferencias más importantes entre los tres taxones.

\section{Clave para las especies de Zygia de la Península de Yucatán}

1. Lámina de los foliolos y venación claramente asimétrica, semilimbo grande con la venación más conspicua y semilimbo más pequeño con la venación menos conspicua; dos o tres nervios secundarios naciendo cerca de la base en un ángulo muy cerrado con relación al nervio principal, dispuestos más o menos paralelamente al margen; flores con cáliz $\leq$ de 1,6 $\mathrm{mm}$ de largo; frutos hasta $3,3 \mathrm{~cm}$ de ancho, ligeramente curvos con semillas imbricadas cuando maduras

Z. latifolia var. latifolia

1. Lámina de los foliolos y venación simétricas; los nervios secundarios todos más o menos iguales y en un ángulo abierto con relación al nervio principal, en la base perpendicular al margen; flores con el cáliz hasta $4,1 \mathrm{~mm}$ de largo; frutos hasta $2,4 \mathrm{~cm}$ de ancho, rectos y cilíndricos o aplanados y entonces enrollándose hasta dar una vuelta o vuelta y media, con semillas nunca imbricadas cuando maduras

2. Hojas de $16-32 \mathrm{~cm}$ de largo; raquis de la pinna de 3,7-17 cm de largo, rara vez reducida a solo $0,5 \mathrm{~cm}$ de largo; foliolos terminales 4-14 cm de largo, márgenes aplanados; cáliz hasta $4,1 \mathrm{~mm}$ de largo; fruto recto..........

2. Hojas de 6-20 cm de largo; raquis de la pinna de 2,5-5,6 cm de largo; foliolos terminales 3-11,5 cm de largo, márgenes ondulados; cáliz hasta 3,3 mm de largo; fruto curvado. Z. recordii

Zygia cognata (Schltdl.) Britton \& Rose, N. Amer. Fl. 23 (1): 39. 1928. - Inga cognata Schltdl., Linnaea 12: 560. 1838. Tipo: México. Veracruz, in regione calida Colipam et littora maris Martio, Schiede 676 (HAL-0071728!, Lectotipo designado por Barneby \& Grimes, 1997). Figs. 1E-H, 2 A-B, 3.

Zygia stevensonii (Standl.) Killip ex Record, Trop. Woods 63: 6. 1940.-Inga stevensonii Standl., Trop. Woods 23: 7. 1930-Pithecellobium stevensonii (Standl.) Standl. \& Steyerm., Publ. Field Mus. Nat. Hist., Bot. Ser. 23 (4): 164. 1944. Tipo: Belice. Corozal, Freshwater Creek, March, 1928, D. Stevenson (II) 65 (holotipo, F!, isotipo, Y).

Árboles 2-10 m de alto; ramas jóvenes glabras; estípulas lanceoladas, 1,5-2,3 mm de largo, persistentes o caedizas. Hojas 16-32 cm de largo, bipinnadas, 6 ó 10 foliolos por hoja; pecíolo circular en sección transversal, glabro, $0,6-2 \mathrm{~cm}$ de largo, glándula terminal, circular o ligeramente rómbica y con un anillo en el borde cuando seco; pinna 3,7-17 cm de largo, pubescente, con una glándula en el ápice, circular o ligeramente rómbica, más pequeña que la glándula del pecíolo; foliolos dimorfos, el basal al menos la mitad del tamaño de los foliolos distales, estos últimos elípticos, elípticos-obovado o elíptico-ovados, 6,5-14,5×2-5, base cuneada, asimétrica y engrosada con aspecto pulviniforme; nerviación penninervia, asimétrica, en la porción más grande, 6-7 pares de nervios secundarios y con un ángulo de más o menos 45 grados y prolongándose hacia el ápice, en la porción más pequeña, con patrón similar menos conspicuo, glabros en ambas caras, ápice agudo o angostamente acuminado, base oblicua, margen aplanado. Inflorescencia espiga $\mathrm{u}$ ocasionalmente tendiendo a un capítulo, $2,3-4,8 \mathrm{~cm}$ de largo, pedúnculo 1,05-1,15 cm de largo, flores 9-17, amarillo pálido, sésiles o con un corto pedúnculo de hasta $1 \mathrm{~mm}$ de largo, todo el eje diminutamente puberulento, una bráctea por flor, triangular, ca. $1 \mathrm{~mm}$ de largo, puberulenta, márgenes ciliados; cáliz tubular, 2,5-4,1 mm de largo, 5 diminutos lóbulos triangulares hasta angostamente triangulares ca. 0,6 mm de largo; corola 4,3-9,8 mm de largo, lóbulos ovados, ca. $1 \mathrm{~mm}$ de largo, glabros; androceo con 29-45 estambres, rojizos, 12,6-17,2 $\mathrm{mm}$ de largo, tubo $6-11,5 \mathrm{~mm}$ de largo, exerto; disco intraestaminal muy reducido; primordio seminal, sésil 1,5-2 mm de largo, glabro; estilo ligeramente más largo que los estambres; estigma escasamente diferenciado. Fruto subsésil, linear en perfil, recto, cilíndrico, 14-20×1,2-2,4 mm, dehiscente a través de la sutura ventral; valvas cartáceas, pubescentes en la superficie externa, suturas escasamente prominentes en el margen semillas 8-16, discoides, sin pleurograma, testa parda.

Material representativo examinado: MÉXICO: Campeche: D. Álvarez E al. 1844 (M, MEXU, MO); E. Martínez E al. 28127 (UCAM). Quintana Roo: E. Cabrera y de H. Cabrera 3448 (BM, MEXU, MO); 6638 (MO); G. Carnevali \& al. 5279 (CICY); R. Duno \& al. 2103 (CICY, MEXU, US); R. Durán E al. 3231 (CICY, MO); S. Escalante 254 (CIQRO); O. Téllez 2564 (CICY, MEXU, MO); O. Téllez y E. Cabrera 3242 (CIQRO, NY), M. Sousa y E. Cabrera 12312 (CICY, K, MEXU, MO); E. Ucán 4028 (CICY, K). Yucatán: C. Chan 6764 (CICY, UCAM).

Comentario taxonómico: Zygia cognata tiene una inflorescencia tipo espiga corta, a veces pareciendo un capítulo. El fruto es cilíndrico, largo y ligeramente curvado. Sin embargo, sí los primordios seminales no fueron fertilizados (e.g. C. Chan 6764, CICY, UCAM), el fruto permanece aplanado dorsiventralmente, pudiendo ser más largos y delgados que el fruto de $Z$. latifolia $(25-30 \mathrm{~cm}$ de largo). Es morfológicamente afín a Z. peckii (B.L. Rob.) Britton \& Rose [Belice (Belice y Toledo), Guatemala (Alta Verapaz, Izabal y Petén) y México (Chiapas y Tabasco)], diferenciándose porque $Z$. peckii tiene los foliolos más anchos y fruto más largo y ancho $(9-15 \times 2,4-4 \mathrm{~cm})$. Carnevali \& al. (2010) reconocen Z. stevensonii en el área de la Península de Yucatán; cabe mencionar que colecciones estudiadas provenientes de Oaxaca (L. Cortes E al. 901, 1986, J.I. Calzada 14856, Sousa \& al. 13055, MEXU) coinciden con la morfología de las poblaciones de la Península de Yucatán, y por ello en el presente estudio se anotaron como Z. cognata. 


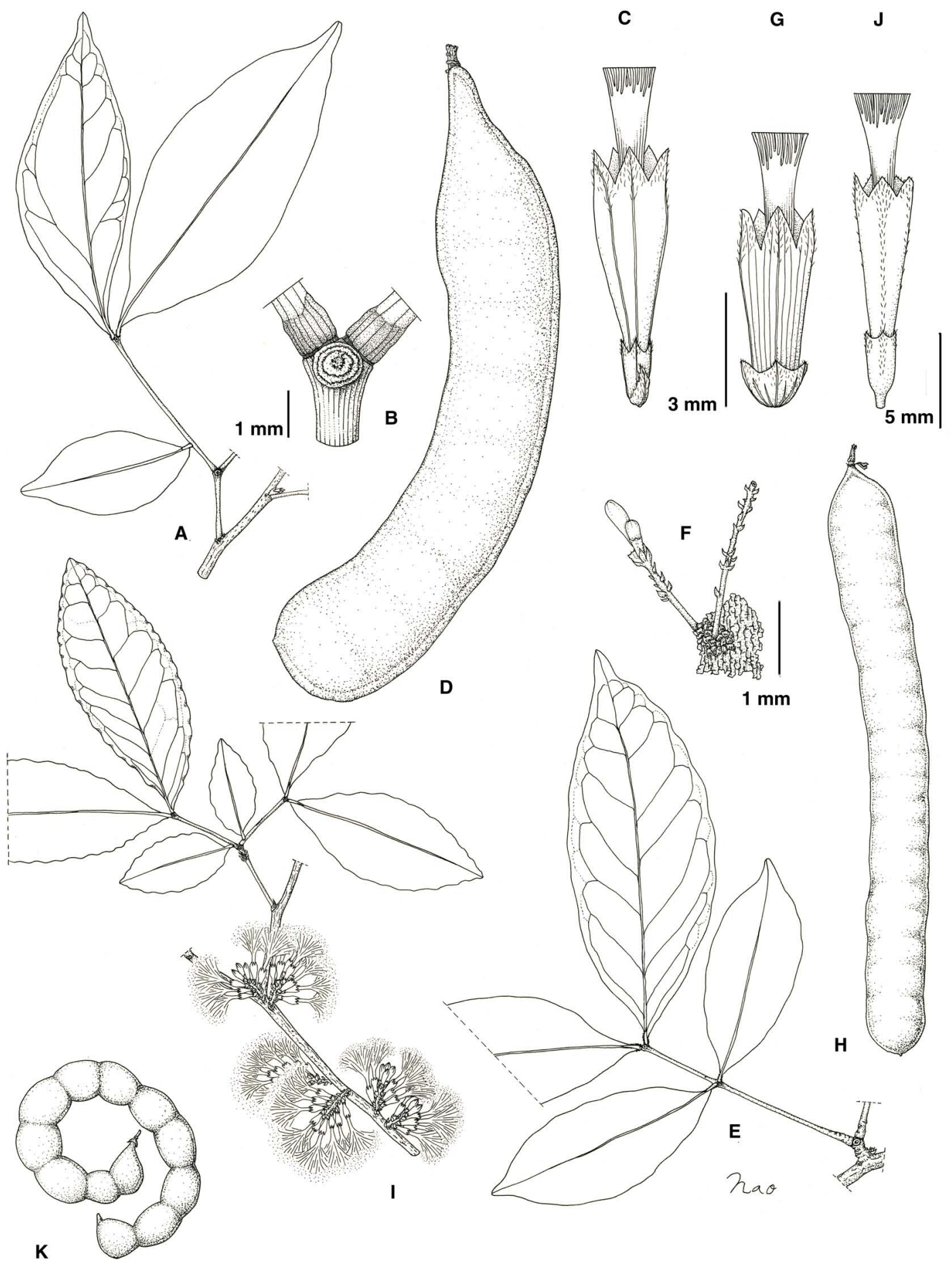

Fig.1. A-D) Zygia latifolia. A) rama mostrando una hoja con una sola pinna; B) glándula peciolar; C) flor completa; D) legumbre. E-H) Z. cognata. E) rama mostrando una hoja y una pinna; F) inflorescencia mostrando caulifloria, brácteas florales y algunas yemas florales; G) flor completa; H) legumbre. I-J) Z. recordii. I) rama mostrando una hoja y un par de pinnas; J) flor completa; K) fruto (A-C Sousa 11759; D-F Campbell 395; E. Sousa y Cabrera 12312; G Gentle 1171; H Hayes 464; I-K, Macqueen 528; J. Álvarez 10486). Dibujo preparado por N. Yasue.

Las muestras y tipos de Christian Julius Wilhelm Schiede fueron depositados principalmente en el herbario de Berlín (B) y en la Universidad de Martin-Luther (HAL). La primera de estas colecciones fue destruida en la Segunda Guerra Mundial. Rupent Barneby estudio en el año 1993 dos ejemplares de Inga cognata depositados en HAL: 0107632 y 0071728; indicando la segunda de ellas como holotipo (Barneby \& Grimes, 1997). En concordancia con el Código Internacional de Nomenclatura (McNeil \& al., 2012) aceptamos como una lectotipificación de facto la realizada por R. Barneby del ejemplar HAL-0071728. 


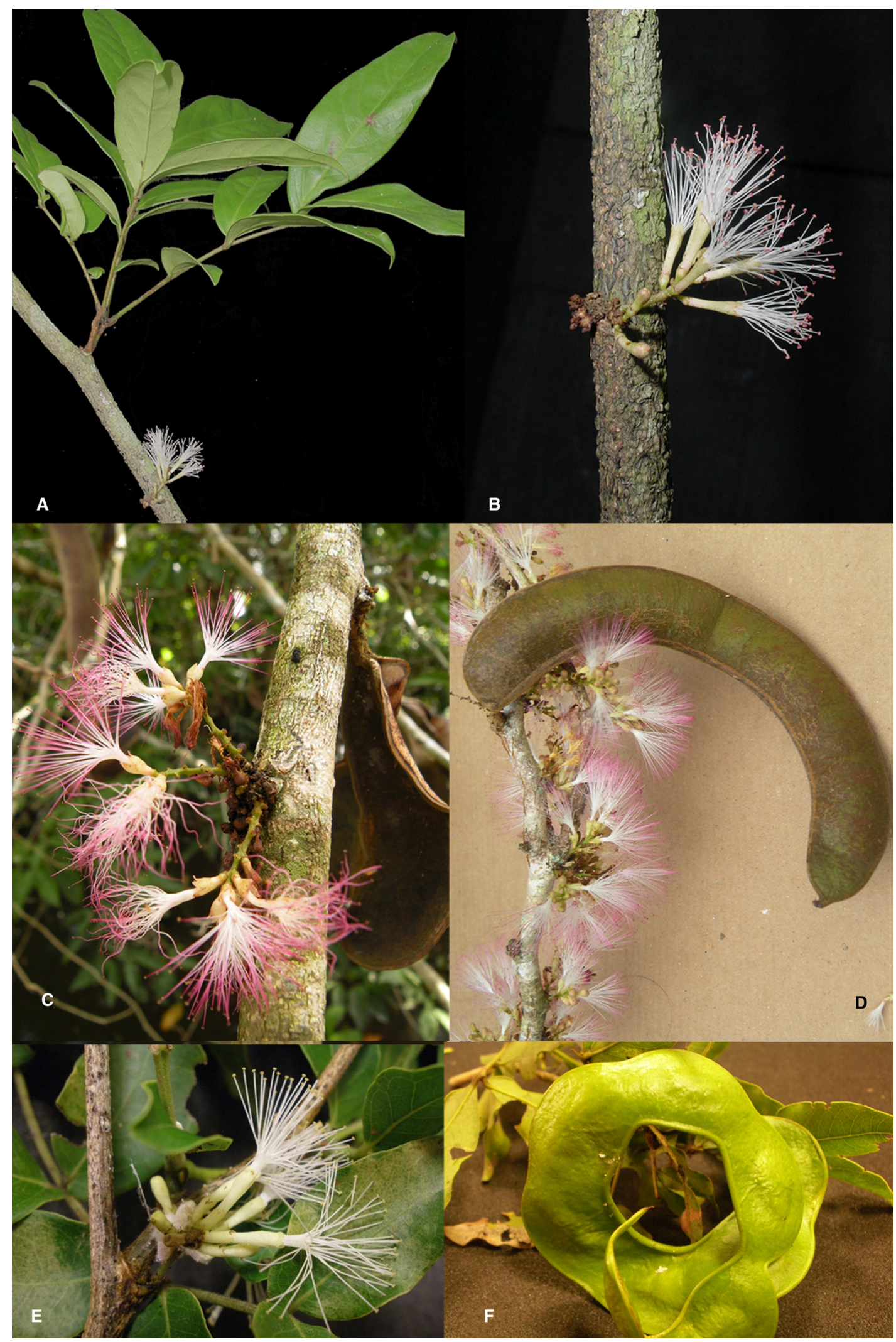

Fig. 2. Zygia en la Península de Yucatán. A y B) Z. cognata cognata; C y D) Z. latifolia; E y F) Z. recordii.

Distribución y ecología: Zygia cognata se distribuye en México (Campeche, Chiapas, Oaxaca, Quintana Roo, Tabasco y Veracruz), en Guatemala (Alta Verapaz) y Belice (Belice).
Habita en la selva mediana subperennifolia y selva alta perennifolia entre 0 y 300 metros sobre el nivel del mar. La colección C. Chan 6764 (CICY, UCAM) se localizó disyuntamente 


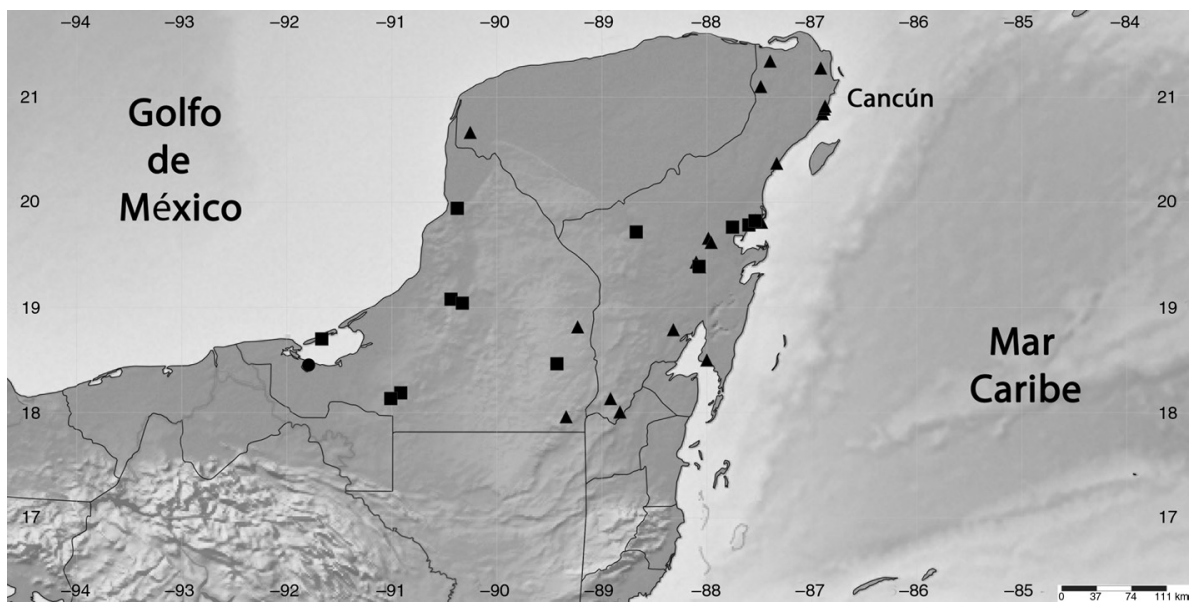

Fig. 3. Distribución de Z. cognata $(\mathbf{\Lambda})$, Z. latifolia $(\bullet)$ y Z. recordii (⿶) en la porción mexicana de la Península de Yucatán.

al noroeste de Yucatán y el área corresponde a una selva baja caducifolia. Zygia cognata florece entre mayo y agosto y fructifica entre junio y enero. Localmente se conoce como "cacaoché", "kakaw che", "kakawché" y "x-chook chê" y en Belice como "Turtle bone" (J.B. Kinloch 17). La madera de esta especie es localmente usada para la construcción.

Zygia latifolia (L.) Faw. \& Rendle var. latifolia, Fl. Jamaica 4: 150. 1920-Mimosa latifolia L., Syst. Nat., ed. 10: 1310. 1759. Tipo: Habita in America [basado en Plumier, Cat. Pl. Amer. 17. 1703]. Figs. 1A-D, 2 C-D, 3.

Arbustos o árboles 4-15 m de alto, excepcionalmente alcanzando $20 \mathrm{~m}$; ramas jóvenes glabras; estípulas triangular-ovadas o deltoides, 0,9-2,2 $\mathrm{mm}$ de largo, persistentes o caducas, pubescente en especial en el ápice. Hojas $9-18,5 \mathrm{~cm}$ de largo, bipinnadas, 6 foliolos por hoja; peciolo circular en sección transversal, puberulento, 0,9-2,1 cm de largo, glándula terminal, circular o ligeramente rómbica con un anillo en el borde cuando seco; pinna $2.5-14 \mathrm{~cm}$ de largo, pubescente, con una glándula en el ápice, circular o ligeramente rómbica, más pequeña que la glándula del pecíolo; foliolos dimorfos, el basal al menos la mitad del tamaño de los foliolos distales, estos últimos elípticos o elíptico-oblongos, 8,6-15,5×2,6-5,9 cm, base atenuada, asimétrica y engrosada con aspecto pulviniforme; nerviación palmado-reticulada, asimétrica, en la porción más grande, 2 nervios secundarios nacen de la base, un tercero y ocasionalmente un cuarto, un poco más arriba, estos más conspicuos que el resto y con un ángulo de más o menos $60^{\circ}$ y prolongadas hacia el ápice, en la porción más pequeña, el patrón similar pero menos conspicuo, glabros en ambas caras, ápice agudo, base ligeramente oblicua, margen aplanado. Inflorescencia espigas $1,1-1,5 \mathrm{~cm}$ de largo, pedúnculo muy reducido, flores 7-10, verdosas y ligeramente rojizas en los ápices antes de la antesis, sésiles, todo el eje puberulento, una bráctea por flor, triangular hasta angostamente triangular, ca. $1 \mathrm{~mm}$ de largo, puberulenta, márgenes ciliados; cáliz tubular, 0,8-1,6 mm de largo, 5 diminutos lóbulos de triangulares a angostamente triangulares, ca. de $0,3 \mathrm{~mm}$ de largo; corola 6-8 mm de largo, lóbulos ovados, glabros o ligeramente pubescentes hacia el ápice; androceo con 27-48 estambres, rojizos, 14-30 $\mathrm{mm}$ de largo, tubo 6,5-9,4 $\mathrm{mm}$ de largo, exerto; disco intraestaminal, $0,3-0,7 \mathrm{~mm}$ o sin disco; primordio seminal sésil, ca. de 1,2-1,5 mm de largo, glabro; estilo ligeramente más largo que los estambres; estigma escasamente diferenciado. Fruto subsésil, linear en perfil, ligeramente curvado, aplanado en sentido dorsoventral, $12 \times 3,2 \mathrm{~cm}$, dehiscente a través de la sutura ventral, puberulento en la superficie externa; valvas leñosas, glabras en la superficie externa, suturas prominentes en el margen; semillas 6-10, uniseriadas, elípticas o casi circulares, $25-30 \times 20-25 \times 5 \mathrm{~mm}$, imbricadas, sin pleurograma, testa de color pardo.

Material examinado: MÉXICO. Campeche: E. Cabrera y H. de Cabrera 14933 (MEXU, MO, NY), E. López Contreras 1110, 1118, 1121 (CICY). Tabasco: M. Sousa \& al. 11759 (K).

Comentario taxonómico: Zygia latifolia es una especie fácil de reconocer con las dos partes de cada foliolo asimétricos (un semilimbo más grande) y dos o tres nervios secundarios naciendo cerca de la base (en el lado más grande los dos o tres nervios más conspicuos). Esta especie ya había sido colectada en el pasado en la Península de Yucatán (E.Cabrera 14933, MEXU, NY) y reportada solo recientemente (Carnevali \& al., 2010). Sosa \& al. (1985) y Durán \& al. (2000) incluyen este nombre en la Península de Yucatán basado en la muestra E. Ucan 760 [que es Pithecellobium unguis-cati (L.) Benth.] Las muestras de Campeche presentan semillas imbricadas a diferencia de lo mencionado por Barneby \& Grimes (1997). Otras especies presentes cerca del límite suroccidental de la Península de Yucatán son Zygia longifolia (Willd.) Britton \& Rose y Zygia peckii. La primera tiene hojas con seis foliolos, nerviación ligeramente asimétrica y la inflorescencia es una espiga corta y las flores tienen el cáliz más pequeño y la relación corola/ cáliz es mayor (2:1-6:1). El fruto es largo, delgado y ligeramente curvado. La segunda especie, tiene hojas con foliolos más grandes y la inflorescencia es un capitulo también con flores más grandes $(6,5-8 \mathrm{~mm})$, el cáliz es grande y la relación corola/cáliz es menor (2:1-4:1). El fruto es más pequeño y ancho $(9-15 \times 3,5 \mathrm{~cm})$.

Distribución y ecología: Zygia latifolia incluye cinco variedades con una distribución amplia desde el sureste de México hasta Panamá, Sur América y las Antillas (Barneby \& Grimes, 1997). Zygia latifolia var. latifolia se localiza en el Sureste 
de México (Chiapas, Campeche, Tabasco y Veracruz), las Antillas (Cuba, Jamaica y Haití y Martinica) y Sur América (Colombia, Venezuela y Brasil). En México crece en zonas inundables: vegetación tipo manglar y bosques ribereños entre 0 y 100 metros sobre el nivel del mar. Ha sido colectada en flor en febrero y de junio hasta noviembre, en fruto de mayo hasta julio. Es una especie común en Veracruz y Tabasco (Golfo de México) y marginalmente en la Península de Yucatán, donde solo habita las planicies inundables de laguna de Términos y Palizada en el estado de Campeche (Fig. 3).

Zygia recordii Britton \& Rose, Trop. Woods 7: 6. 1926Pithecellobium recordii (Britton \& Rose) Standl., Publ. Mus. Nat. Hist., Bot. Ser. 4 (8): 212. 1929, Tipo: Belice. Orange Walk, Samuel J. Record along the bank of New River, near Guinea Grass, Jan. 29, 1926 (holotipos, US!). Figs. 1I-K, 2 E-F, 3.

Pithecellobium disciferum Lundell, Contr. Univ. Michigan Herb. 6: 27-28. 1941. Tipo: México. Campeche, Vapor east of Palizada, July 25 to 28, 1939, E. Matuda 3908 (holotipo, $\mathrm{MICH}$ !).

Arbustos o árboles 3-11 (-15) m de alto; ramas jóvenes circular en sección transversal y puberulentas hasta glabrescentes; estípulas lanceoladas, 0,5-2 mm de largo, persistentes o caedizas. Hojas 6-16 cm de largo; bipinnada, $6(-10)$ foliolos por hoja total; pecíolo circular en sección transversal, puberulento, $0,1-0,5 \mathrm{~cm}$ de largo, glándula terminal, circular o ligeramente rómbica con un anillo en el borde cuando seco; pinna 2,5-5,6 cm de largo, pubescente, con una glándula en el ápice, circular o ligeramente rómbica, más pequeña que la glándula del pecíolo; raquis de las pinnas $1-6 \mathrm{~cm}$ de largo, puberulentas, glándula terminal más pequeña que la de los pecíolos; foliolos dimórficos, el basal al menos la mitad del tamaño de los foliolos distales, estos últimos elípticos, rara vez elíptico ovado, $3-11,5 \times 1,8-3,5 \mathrm{~cm}$, ápice agudo, base atenuada, asimétrica y engrosada con aspecto pulviniforme; nerviación penninervia, asimétrica, en la porción más grande, 5-14 pares nervios con un ángulo más o menos $45^{\circ}$ grados y uniéndose cerca del margen, glabro en ambas caras, rara vez pubescente, ápice agudo, ocasionalmente retuso, base atenuada, margen conspicuamente ondulado. Inflorescencia capítulo y con el tiempo una diminuta espiga con un receptáculo de $3 \mathrm{~mm}$ de largo, 1,5-2,8 cm de largo, pedúnculo 4-6 $\mathrm{mm}$ de largo, flores 11-18, amarillo pálido, sésiles, todo el eje puberulento, una bráctea por flor, triangular hasta angostamente triangular, ca. $1 \mathrm{~mm}$ de largo, puberulenta con los márgenes ciliados; cáliz tubular, 2,5-3,3 mm de largo, 5 diminutos lóbulos triangulares hasta angostamente triangulares de ca. de $0,4 \mathrm{~mm}$ de largo; corola 5,3-8,5 mm de largo, lóbulos ovados, ca. de $0,4 \mathrm{~mm}$ de largo, glabros o ligeramente pubescentes hacia el ápice; androceo con 30-44 estambres, amarillo pálido, 12-20 mm de largo, tubo 7,8-13 $\mathrm{mm}$ de largo, exerto; disco intraestaminal $0,5 \mathrm{~mm}$ de largo, glabro; primordio seminal sésil, ca. $2 \mathrm{~mm}$ de alto, glabro; estilo ligeramente más largo que los estambres; estigma escasamente diferenciado. Fruto subsésil, linear en perfil, curvado, $12-23 \times 1-1,5 \mathrm{~cm}$, dehiscente a través de la sutura ventral, glabro en la superficie externa, valvas cartáceas, suturas escasamente prominentes en el margen; semillas, 7-14, uniseriadas, ampliamente elípticas, $15 \times 12 \times 13,5 \mathrm{~mm}$, sin pleurogramas, testa parda.

Comentario taxonómico: Barneby \& Grimes (1997) incluyen a $Z$. scopulina (Brandegee) Britton \& Rose, Z. recordii y $Z$. discifera L. Rico como sinónimos. Los materiales tipos de todas estas especies muestran diferencias morfológicas y distribuciones geográficas suficientes para reconocer a $Z$. recordii como una especie distinta con foliolos distales hasta $11,5 \mathrm{~cm}$ de largo (hasta $6,2 \mathrm{~cm}$ en $Z$. conzatti), cáliz de hasta 2,3-3,3 mm de largo (menos de $1 \mathrm{~mm}$ en $Z$. conzattii), la relación corola/cáliz es 1: 1,8-2,6 (1: 5-5,5 en $Z$. conzattii). Por último, $Z$. recordii tiene frutos aplanados dando una o una vuelta y media ( $Z$. conzattii tiene el fruto aplanado ligeramente curvado).

En la Península de Yucatán, Z. recordii presenta vegetativamente dos formas, la típica con solo 6 foliolos pequeños por hojas y otra forma con 5 ó 10 foliolos más grandes [e.g. G. Carnevali 4494 (CICY; UCAM), C. Gutiérrez Báez 5785 (UCAM), P. Zamora 5096, 5682 (UCAM)], en ambos casos los foliolos muestran los márgenes ondulados típicos de la especie.

Distribución y ecología: Zygia recordii se distribuye en Chiapas, Campeche, Oaxaca (Tuxtepec), Quintana Roo, Tabasco, Veracruz y en Centroamérica hasta Honduras (Fig. 3). En la Península de Yucatán mexicana habita en selva mediana subcaducifolia y selva mediana subperenifolia entre 0 y 100 metros sobre el nivel del mar y en otras áreas de México y Centro América hasta los 500 metros. Florece entre marzo y agosto y fructifica entre mayo y octubre. En Belice se conoce como "Turtle bone" (nombre general para varios congéneres en esta área). Por su parte, Zygia conzattii se limita a la costa Pacifico de México en los estados de Oaxaca y Guerrero.

Material seleccionado examinado: MEXICO: Campeche: D. Álvarez 1278 (K, MEXU, UCAM), 8969 (NY), 10259 (K); E. Cabrera y H. de Cabrera 3263 (CICY), H. de Cabrera 14103 (CIQRO, MEXU, US), 14495 (MO); G. Carnevali E al. 4494 (CICY, UCAM), 6045, 6065, 6108 (CICY), 6149 (CICY, UCAM); C. Chan 3784 (K); C. Gutiérrez Báez 5785 (CICY, UCAM); C. Gutiérrez Báez 7280 (UCAM); E. Martínez E al. 28010, 28013 (MEXU, UCAM), 28145-A (UCAM), 28356 (CICY, MEXU, NY, UCAM). Quintana Roo: D. Álvarez \& al. 10486 (K), 10492 (K, NY), R. Durán y I. Olmsted 398 (CICY), 476 (CICY); C.L. Lundell, 1497 (MICH); O. Téllez y E. Cabrera 3090 (CICY; CIQRO), 3100 (CICY, CIQRO, NY). Yucatán: O.G. Enriquez 624 (US).

\section{AGRADECIMIENTOS}

Los autores agradecen a los curadores de los siguientes herbarios A, AAU, CICY, ENCB, F, G, HAL, MEXU, MICH, M, MO, NY, CIQRO PH, UCAM, US, especialmente al Dr. Laurence J. Dorr (US) y Stella Silva (NY). A la Dra. Ivon Ramírez (CICY) por la lectura crítica del texto, y a Amilcar Castillo y Silvia Hernández en el herbario CICY y Naoko Yasue por elaborar la ilustración botánica. La investigación fue financiada por el proyecto de ciencia básica 81799 de CONACYT y el proyecto 1039300012 de CICY. Asimismo el segundo autor agradece al Missouri Botanical Garden y la beca Alwyn H. Gentry (2013) por la oportunidad de visitar el herbario MO en los meses de abril y mayo del año 2014. 


\section{REFERENCIAS BIBLIOGRÁFICAS}

Arellano-Rodríguez, J.A., Flores Guido, J.S., Tun Garrido, J. \& Cruz Bojórquez, M.M. 2003. Nomenclatura, forma de vida, uso, manejo y distribución de las especies vegetales de la Península de Yucatán. Etnoflora Yucatanense 20: 1-815.

Barneby, R.C. \& Grimes, J.W. 1997. Silk tree, guanacaste, monkey's earring: a generic system for the synandrous Mimosaceae of the Americas. Part II. Pithecellobium, Cojoba, and Zygia. Memoirs of the New York Botanical Garden 74: 1-149.

Browne, P. 1756. The Civil and Natural History of Jamaica. London. 503 pp. Bentham, G. 1844. Note on Mimoseae with a synopsis of species. Tribe III. Acacieae. London Journal of Botany 3: 195-226.

Bentham, G. 1875. Revision of the suborder Mimoseae. Transactions of the Linnaean Society London 30: 335-664. pls. LXVI-LXX. http://dx.doi. org/10.1111/j.1096-3642.1875.tb00005.x

Brown, G.K., Murphy, D.J., Miller, J.T \& Ladiges, P.Y. 2008. Acacia s.str. and its Relationships Among Tropical Legume, Tribe Ingeae (Leguminosae: Mimosoideae). Systematic Botany 33: 739-751. http:// dx.doi.org/10.1600/036364408786500136

Britton, N.L. \& Rose, J.N. 1928. Mimosaceae. North American Flora. 23: 1-76.

Carnevali Fernández-Concha, G., Tapia-Muñoz, J.L., Duno de Stefano, R. \& Ramírez, I.M. 2010. Flora Ilustrada de la Península de Yucatán: Listado Florístico. CICY, A.C., Mérida. 326 pp.

Durán, R., Campos, G., Trejo, J.C., Simá, P., May Pat, F. \& Juan Qui, M. 2000. Listado Florístico de la Península de Yucatán. CICY, A.C., Mérida. 259 pp.

Fawcett, W. \& Rendle, A.B. 1920. Flora of Jamaica containing descriptions of the flowering plants known from the island. Vol. 4 Dicotyledons Families Leguminosae to Callictrichaceae. British Museum, Londres. 369 pp.
Linnaeus, C. 1759. Volume 2. Regnum Vegetabile. Systema Naturae (10th ed.). Laurentius Salvius. Stockholm, 1384 pp.

McNeill, J., Barrie, F.R., Buck, W.R., Demoulin, V., Greuter, W., Hawksworth, D.L., Herendeen, P.S., Knapp, S., Marhold, K., Prado, J., Prud'homme van Reine, W.F., Smith, G.F., Wiersema, J.H. y Turland, N.J. 2012 International Code of Nomenclature for Algae, Fungi, and Plants (Melbourne Code). Regnum Vegetabile 154. Königstein: Koeltz Scientific Books. 492 pp.

Nielsen, I. 1981. Ingeae. In: Polhill, R.M. \& Raven, P.H. (eds.), Advance in Legume Systematics Part 1. Royal Botanic Gardens, Kew, Richmond. pp. 173-193.

Sosa, V., Salvador Flores, J., Rico-Gray, V., Lira, R. \& Ortíz, J.J. 1985. Lista Florística y Sinonímia Maya. In: Etnoflora Yucatanense 1: 1-225.

Rodrigues de Souza, E., Lewis, G.P., Forest, F., Schnadelbach, A.S., van den Berg, C. \& Paganucci de Queiroz. L. 2013. Phylogeny Calliandra (Leguminosae: Mimosoideae) based on nuclear and plastid molecular markers. Taxon 62: 1201-1220. http://dx.doi.org/10.12705/626.2

Shorthouse, D.P. 2010. SimpleMappr, an online tool to produce publicationquality point maps. [Retrieved from http://www.simplemappr.net. accedido: 15 de Marzo de 2013].

Thiers, B. 2010 Index herbariorum: A global directory of public herbaria and associated staff. New York Botanical Garden's Virtual Herbarium, New York. http://sweetgum.nybg.org/ih/ (continuamente actualizado; accedido 20 de Enero de 2014).

Woodson, R.E., Jr. \& Schery, R.W. 1950. Flora of Panama. Part V, Fascicle 2 (Resedaceae to Leguminosae, in part). Annals of the Missouri Botanical Garden 37: 121-314. http://dx.doi.org/10.2307/2394414

Associate Editor: Manuel de la Estrella Received: 27-V-2015 Accepted: 9-X-2015 